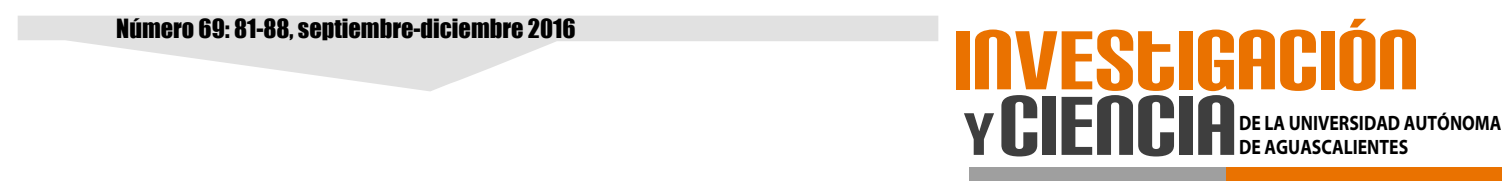

\title{
El mercado de trabajo mexicano, las dificultades de la informalidad
}

\author{
The Mexican labor market, the difficulties of informality
}

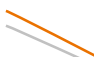

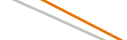

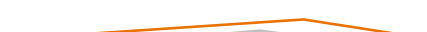

\section{RESUMEN}

Este artículo presenta una revisión de indicadores laborales, económicos y demográficos, que dan cuenta de las transformaciones del mercado laboral en los últimos años. Las actividades informales y los micronegocios resultan ser principales las características del mercado de trabajo mexicano.
\end{abstract}

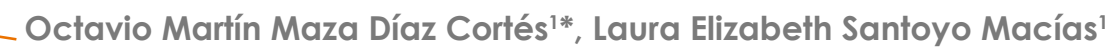

Maza Díaz Cortés, O. M. Santoyo Macías, L. E. El mercado de trabajo mexicano, las dificultades de la informalidad. Investigación y Ciencia de la Universidad Autónoma de Aguascalientes. Número 69: 81-88, septiembre-diciembre 2016.

\section{ABSTRACT}

In this article, a review of the demographic indicators, labor indicators and economic indicators are presented for the purpose to acknowledge the changes that have occurred in the Mexican labour market, in order to prove the need that the public policies have to focus in the work that is developed in smaller economic units.

\section{INTRODUCCIÓN}

El desarrollo de las condiciones laborales en países como México ha puesto en evidencia una clara tendencia en la trasformación del mercado laboral, lo que ha dado lugar a una multiplicidad de formas de trabajo, mismas que han sido desatendidas por los investigadores y, lo que es más grave, por el Estado. Esta condición se explica en el hecho de que representan un gran reto, tanto para los estudios

Palabras clave: mercado de trabajo, informalidad, precariedad.

Keywords: work, precariousness, informality.

Recibido: 18 de junio de 2015, aceptado: 16 de junio de 2016

Departamento de Sociología, Centro de Ciencias Sociales y Humanidades, Universidad Autónoma de Aguascalientes.

* Autor para correspondencia: octaviomazadc@gmail.com laborales como para la implementación de las políticas públicas. Se trata de condiciones de trabajo que presentan características que lo hacen diferente al estándar o típico y para el Estado resultan complejas en su control y vigilancia, al tiempo que solucionan los problemas de empleo que afectan a gran parte de la población. En este artículo se presenta una revisión de algunos indicadores que cubren el periodo comprendido desde 2008 hasta 2013 (para facilitar la comparabilidad). El objetivo planteado fue dar cuenta de las condiciones del mercado de trabajo mexicano, con especial atención en las dimensiones de informalidad, la ocupación en los micronegocios y el índice de tendencia laboral de la pobreza. Dichos indicadores permiten reconocer al trabajo precarizado como uno de los factores que favorece el incremento de la pobreza.

\section{Contexto del mercado laboral en México}

En las últimas décadas en México se han generado cambios sociodemográficos, políticos y económicos que han trastocado la dinámica del mercado laboral. Uno de los que consideramos ha modificado mayormente es el aumento de la población en edad de trabajar, resultado del bono demográfico que presentó el país apenas hace un par de años. Esta condición representaba una ventaja por la cantidad disponible de mano de obra con la que contaba México. La población resultado del bono demográfico se caracterizó por los altos niveles de capacitación y mayores niveles de escolaridad, lo cual la convertía en una población altamente productiva y competitiva.

Otro de los factores a considerar y que ha impactado el mercado laboral son los cambios estructurales que se han gestado dentro de él, los cuales, en términos generales, se han traducido en 
la precarización del mercado de trabajo (Pacheco Gómez Muñoz et al., 2011) que se visibilizan a través de nuevas formas de contratación que adquieren diversos matices en lo que refiere a la duración y extensión del trabajo; asimismo, la configuración de las relaciones salariales y las jornadas laborales.

Las modificaciones que ha experimentado el mercado de trabajo mexicano generan cambios que se traducen en empleos desprotegidos, los cuales incentivan la incorporación de la mano de obra a puestos de trabajo caracterizados por la desprotección social y laboral, lo que da lugar a una mayor participación dentro del sector informal. Del mismo modo, el desempleo y subempleo se convierten en alternativas propias para la mano de obra mexicana.

En las líneas siguientes se presenta la revisión de algunos indicadores de orden económico y demográfico que ayudarán a explicar las condiciones de cambio que se han generado en el mercado laboral mexicano.

Población ocupada y condiciones en el empleo. En México, una de las principales fuentes de información que muestra estadísticas que permiten explicar las condiciones laborales de los mexicanos es la Encuesta Nacional de Ocupación y Empleo (ENOE). De acuerdo a la información generada, la población ocupada en México desde 2008 a la fecha ha llegado a representar en promedio $95 \%$ de la población económicamente activa (PEA) y solo $5 \%$ se encuentra en condiciones de desocupación (INEGI, 2013).
Es importante señalar que la población ocupada se encuentra distribuida en diversas posiciones de ocupación, ello genera que adquieran condiciones de trabajo diversas, lo cual convierte al mercado laboral en un espacio heterogéneo (Pacheco Gómez Muñoz, 2004).

La ENOE muestra que con respecto a el posicionamiento que tenían en el empleo los trabajadores para el tercer trimestre de 2013, 67.2\% eran trabajadores subordinados y remunerados (como se observa en la Figura 1, donde el mayor porcentaje de ellos refiere a los asalariados con 93.8\%), siguen los cuenta propia (22.1\%), los no remunerados con $6.2 \%$, y tan solo $4.6 \%$ de la población ocupada son empleadores.

Es primordial reflexionar que solo una parte de la población ocupada obtiene protección en términos de garantías laborales, lo que permite suponer que son prácticamente los trabajadores subordinados y remunerados; mientras que los que trabajan por cuenta propia, los trabajadores no remunerados y los empleadores generalmente se ven limitados y carentes de protección, producto de la posición que tienen en la ocupación.

Otro dato que merece atención y permite captar algunas condiciones del mercado de trabajo mexicano es la participación de la población ocupada de acuerdo al tamaño de la unidad económica. En México, los ocupados representan una participación de $47.5 \%$ en los micronegocios, especialmente en aquellos que no cuentan con un establecimiento (véase la Figura 2), siguen los

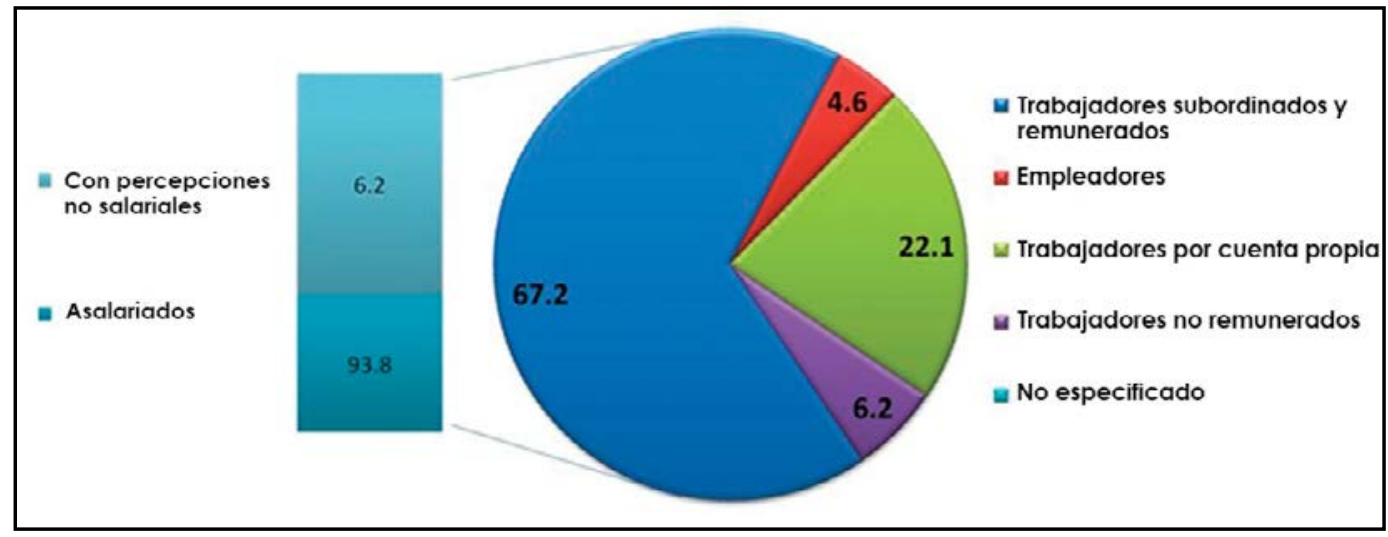

Figura 1. Porcentaje de población ocupada según el posicionanmiento en la actividad, tercer trimestre de 2013. Elaboración propia con datos del tercer trimestre de la ENOE (INEGI, 2013). 


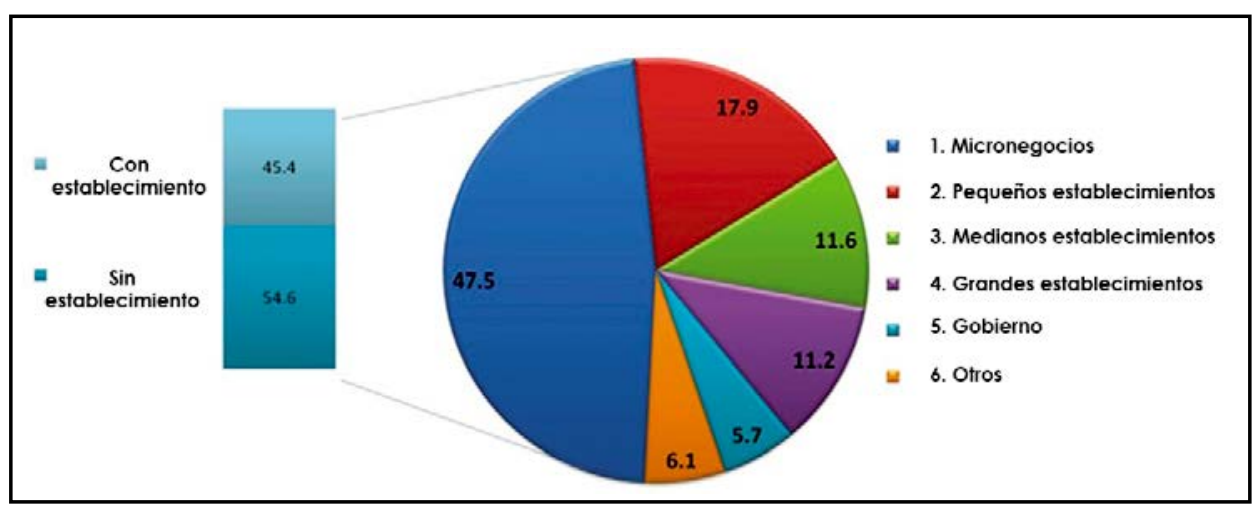

Figura 2. Porcentaje de población ocupada en ámbito no agropecuario por tamaño de la unidad económica. Elaboración propia con datos del tercer trimestre de la ENOE (INEGI, 2013).

pequeños establecimientos (17.9\%), posteriormente, los medianos y grandes establecimientos $111.6 \%$ y $11.2 \%$, respectivamente) y finalmente, un poco más del $11 \%$, está en el sector gobierno u otros. Se debe de poner atención en que en los últimos años un poco más de las tres cuartas partes de la población se encuentran ocupadas en las micro, pequeñas y medianas empresas.

El incremento de participación de la población ocupada en unidades económicas de menor tamaño se explica por las estrategias de la población para acceder al mercado de trabajo, cuando el resto de las alternativas de ocupación se constriñen y limitan la participación de la población.

Otro de los aspectos que resultan relevantes para comprender el mercado de trabajo son las estadísticas que describen la informalidad desde el sector de los hogares ${ }^{2}$. En México, de acuerdo a la ENOE, $34.6 \%$ de la población ocupada se concentra en dicho sector, de la cual $76.7 \%$ se encuentra participando en la informalidad (véase la Figura 3). Se asume que este porcentaje del sector de los hogares participa esencialmente en los micronegocios, conformado por un tipo de unidad económica que es caracterizado por la desprotección y falta de garantías laborales. A lo anterior se debe añadir $49.6 \%$ de personas que están laborando en empresas y negocios y tan sólo $13.5 \%$ trabaja en las instituciones (véase la Figura 3).

La densidad de la población ocupada en los sectores de actividad económica son otro referente para identificar las condiciones del mercado laboral mexicano. De acuerdo al posicionamiento de la ocupación (especialmente los trabajadores subordinados y remunerados) la información proveniente de la ENOE muestra que durante el periodo comprendido desde 2008 hasta 2013, un poco más de $60 \%$ se concentró en el sector terciario, alrededor de $20 \%$ en el sector secundario y menos de $10 \%$ en el primario. Lo anterior permite explicar
2 Definen a la informalidad como aquella que contiene "las unidades económicas no constituidas en sociedad, en el sector informal más las que realizan trabajo doméstico remunerado o agricultura de subsistencia" (INEGI, 2013). Es decir, que se incluyen "todas aquellas actividades económicas de mercado que operan a partir de los recursos de los hogares, pero sin constituirse como empresas con una situación identificable e independiente de esos hogares (INEGI, 2013). El criterio operativo para determinar la situación no independiente de las unidades de producción con respecto al hogar, lo da la ausencia de prácticas contables convencionales, susceptibles de culminar en un balance de activos y pasivos: el que no se realicen quiere decir que no hay una distinción entre el patrimonio del hogar y el de la empresa, ni tampoco hay una distinción entre los flujos de gasto del negocio de los del hogar (por ejemplo: gastos de electricidad y teléfono, uso de vehículo, etc.) (INEGI, 2013).

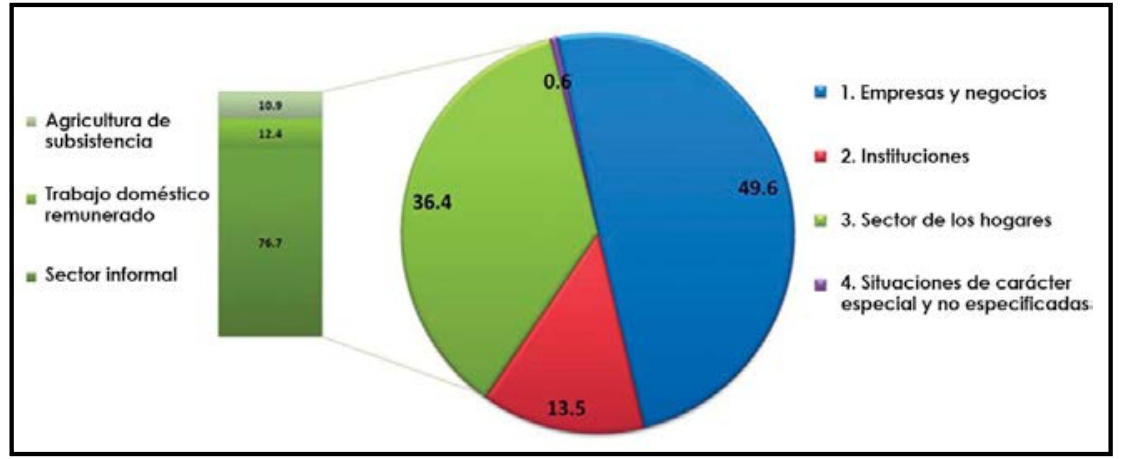

Figura 3. Porcentaje de población ocupada en ámbito no agropecuario por tipo de unidad económica. Elaboración propia con datos del tercer trimestre de la ENOE (INEGI, 2013). 
cómo las actividades económicas se concentran preferentemente en el sector servicios, donde están ausentes la seguridad social y predominan las precarias condiciones laborales. Es importante señalar que son en este tipo de sectores donde predominan los establecimientos informales, los cuales se ven favorecidos debido a que requieren menores niveles de inversión para la constitución de puestos de trabajo.

Con respecto a la adscripción de trabajadores con prestaciones sociales, como las que ofrece el Instituto Mexicano del Seguro Social (IMSS) en términos de servicios médicos, la población beneficiaria en el periodo comprendido desde 2008 hasta 2013 se incrementó. Lo anterior, como resultado de los aumentos de trabajadores con contrato temporal o eventuales (de 11 a 14\%). Este tipo de condicionamientos contractuales aseguran al trabajador por un periodo determinado la seguridad médica; sin embargo, refleja la nula protección a largo plazo. En este mismo sentido, de acuerdo a las cifras sobre trabajadores permanentes adscritos al IMSS, las estadísticas muestran una disminución del número de trabajadores de 89 a $86 \%$. Lo anterior permite inferir cómo las nuevas modalidades de contratación están generando modificación en el mercado laboral, de tal forma que los empleos no logren dar garantías en prestaciones sociales básicas a largo plazo. En este sentido, De la Garza Toledo y Neffa afirman que "el porcentaje de trabajadores con contrato indeterminado ha tendido a disminuir en los primeros años de este siglo y el de los trabajadores sin prestaciones aumentó y luego se estabilizó en el nivel del año 2000" (2010: 65)

La ENOE también se encuentra encargada del cálculo de algunos indicadores laborales, mismos que sirven para explicar el contexto del país en términos de ocupación, desocupación y subocupación. La tasa de participación ${ }^{3}$ para México en el período comprendido desde 2008 hasta 2013 tuvo un crecimiento significativo. Para el país, el incremento de esta tasa implica que existe una mayor cantidad de personas en condiciones de laborar, las cuales se representan por la población económicamente activa.

3 La tasa de participación es el porcentaje que representa la población económicamente activa (PEA) con respecto a la de 14 y más años de edad (INEGI, 2013).

4 Indica el porcentaje de población ocupada que tiene la necesidad y disponibilidad de ofertar más tiempo de trabajo de lo que su ocupación actual le permite.
En lo referente a la tasa de desocupación en el país, el número de personas que estuvieron en condiciones de desempleo aumentó de 2008 a 2013 (de 3.9 a 4.9\%). En términos absolutos, para 2013, lo anterior representa un total de 2.56 millones de personas desempleadas, a las cuales se deben sumar los desempleados desalentados, quienes en el contexto del mercado laboral mexicano se adhieren a los indicadores relativos a la calidad del empleo y la falta del mismo. Desafortunadamente, en el país no se han logrado implementar políticas públicas de protección a los trabajadores en condiciones de desocupación, tal como el seguro de desempleo, que logre dar garantía del sustento y una buena calidad de vida para dicha población.

Ante las condiciones laborales que ofrece el mercado laboral mexicano, existe otro indicador que evidencia la necesidad de los trabajadores de emplearse más horas de trabajo de las que tienen actualmente: la tasa de subocupación ${ }^{4}$. Durante el lapso de 2008 a 2013, la población subocupada se ha incrementado de $6.87 \%$ a $8.35 \%$. Ante las condiciones económicas del país, las personas se encuentran en búsqueda constante de otro tipo de actividades para emplearse y contar con ingresos adicionales. Un ejemplo de lo anterior se explica por la cantidad de personas que participan dentro del sector informal, la mayoría de ellos presentes en los micronegocios o actividades que se realizan en jornadas que ocupan los espacios de otra, como podrían ser las ventas multinivel (Maza Díaz Cortés, 2011).

Los micronegocios: una opción para la ocupación. La información presentada anteriormente muestra que una de las opciones para emplearse ante las condiciones

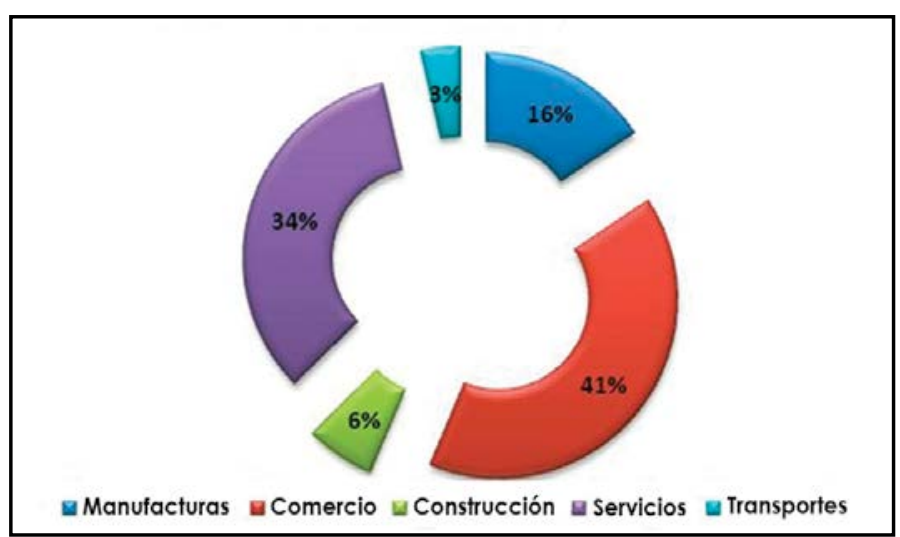

Figura 4. Porcentaje de micronegocios por sector de actividad económica. Elaboración propia con datos de la ENAMIN (INEGl, 2012). 
del mercado laboral mexicano son los micronegocios. Debido a las malas condiciones económicas de las últimas décadas, la falta de oferta de mano de obra, las precarias condiciones laborales y el incremento del desempleo, la población se obliga a buscar nuevos mecanismos para la obtención de ingresos, que permitan la subsistencia de la vida diaria, donde una forma particular se genera a través de la creación de pequeños emprendimientos, también conocidos como micronegocios. Cabe aclarar que de acuerdo a los datos presentados, en la actualidad las microempresas resultan ser la principal fuente de ingresos de una gran parte de la población en el país.

La Encuesta Nacional de Micronegocios (ENAMIN) define a la microempresa como el conjunto de unidades productivas en las que laboran hasta cinco personas (seis incluido el propietario) y solo en el caso de las manufacturas podrán ser hasta 15 personas (16 incluido el dueño). Estas microunidades productivas incluyen también a los trabajadores por cuenta propia (o autoempleados) (INEGI, 2012). De acuerdo con los datos de la ENAMIN 2012 en México se ocupan 9,192,490 personas en los micronegocios, de los cuales $84.49 \%$ son trabajadores por su cuenta y $15.51 \%$ empleadores (INEGI, 2012).

En el país, este tipo de negocios fue promovido como una estrategia para ocupar a una gran parte de la población; de esta forma se incentivaba el autoempleo, se otorgaban subsidios para la creación de microempresas donde se tenía como pretensión ocupar a los miembros de la familia en las llamadas microempresas de tipo familiar, las cuales absorbían la mano de obra y, en la mayoría de los casos, los miembros de la familia no recibían remuneración por el trabajo.

La población trabajadora de los micronegocios principalmente se ocupa en empresas dedicadas al comercio en $41 \%$, otro $34 \%$ a servicios y $16 \%$ a las manufacturas, tan solo $6 \%$ a la construcción y $3 \%$ a transportes (véase la Figura 4). Es importante mencionar que en $82.59 \%$ de los micronegocios, el principal cliente es el público (INEGI, 2012). Este tipo de diseño de negocios tiende a mostrar poca estabilidad por el tipo de clientes a los que pueden acceder. Se debe de tener claro que son las microempresas las que carecen de nulas oportunidades de acceso a seguridad social, bajos o nulos salarios y pocas oportunidades de crecimiento y desarrollo; lo cual reduce las condiciones de bienestar de la población que se encuentra empleada en este tipo de negocios. Además de considerar que aspectos estructurales de la economía mexicana determinan la baja productividad y competitividad de los micronegocios (Cuevas Rodríguez et al., 2005).

Salarios e Índice de tendencia laboral de la pobreza (ITLP) CONEVAL. El índice de la tendencia laboral de la pobreza (ITLP) es un indicador que se presentó a partir de 2005 por el Consejo Nacional de Evaluación de la Política de Desarrollo Social (CONEVAL). Dicho índice refleja las condiciones del mercado laboral mexicano, donde de manera trimestra presenta la tendencia de la proporción de personas que no pueden adquirir la canasta alimentaria con el ingreso de su trabajo, lo cual significa que permite a corto plazo mostrar la situación del ingreso laboral de las familias en relación con el precio de la canasta básica.

Si el índice aumenta, significa que aumenta la proporción de personas que no puede adquirir una canasta alimentaria con su ingreso laboral. Para términos de este análisis, se comparó el ITLP con el salario mínimo, los cuales se encuentran relacionados de manera inversa; de esta forma, cuando el salario aumenta, el ITLP disminuye, y cuando hay una caída del salario, el ITLP aumenta (CONEVAL, s. f.).

Los datos del salario mínimo real en el país muestran que no ha existido un crecimiento significativo en el lapso estudiado de 2008 a 2013, el salario ha oscilado de los 57.54 pesos en 2008 a los 57.71 pesos en 2013, aunque durante el año 2009 fue donde hubo un salario menor (56.67 pesos). Lo anterior puede ser explicado debido a que el salario mínimo real no tuvo un crecimiento significativo, por lo cual la población tuvo menor capacidad de compra, lo que provocó un incremento en el indicador del ITLP (véase la Figura 5).

Ante la tendencia al aumento de los empleos con salarios insuficientes la población busca realizar actividades adicionales que le genere un ingreso extra, con la finalidad de lograr la subsistencia, e inclusive en algunos casos, ante la falta de un salario y la nula posibilidad de tener un trabajo remunerado, las personas se emplean en trabajos no protegidos fuera del sector formal. Al elaborar un análisis confrontando el salario mínimo y el ITLP, en México la situación del mercado laboral expresa la existencia de una mayor cantidad de personas a las que les resulta insuficiente el ingreso que perciben producto de su trabajo, ello permite afirmar que las personas tienden a emplearse en actividades informales que modifican la dinámica y estructura del mercado laboral mexicano. 


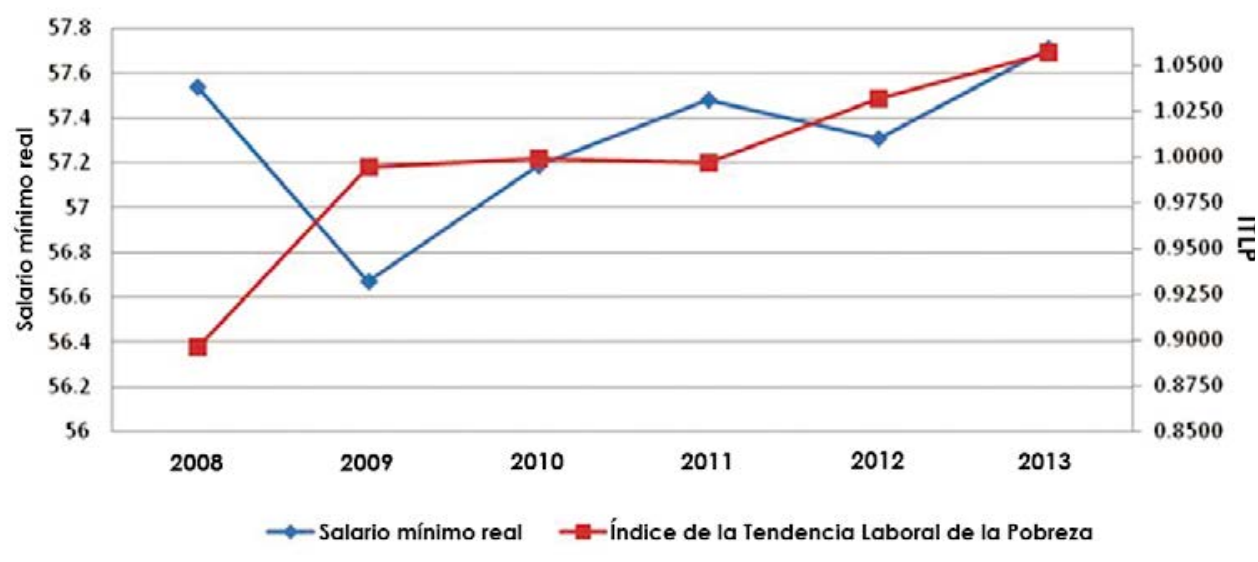

Figura 5. Salario mínimo real e índice de tendencia laboral de la pobreza de México de 2008 a 2013. Elaboración propia con datos de INEGI y CONEVAL (2013).

Informalidad. Tal como se presentaron anteriormente los indicadores de participación de la población ocupada dentro del sector informal; para México, la informalidad representa otro elemento que pone en evidencia las condiciones del mercado de trabajo del país. Se contabiliza dentro de las cuentas nacionales, ya que es un sector fundamental en la economía del país, justificado por la cantidad de personas que se encuentran empleadas, el número de unidades económicas existentes y los ingresos que se generan en este sector.

Las condiciones de ocupación en el sector informal han sido consideradas como un signo de dinamismo del empresario popular. En palabras de Hart, la informalidad es "como una instancia en que la gente retoma en sus propias manos parte del poder económico que trataron de negar los agentes centralizados" (1990: 58). Es importante señalar que este aspecto resulta un tema problemático para el control del Estado y una competencia fuerte para el empresariado establecido. El INEGI, con la información proveniente de la Encuesta Nacional de Ocupación y Empleo (ENOE) hace el cálculo de dos indicadores que permiten ver las condiciones de la informalidad en el país: la tasa de informalidad laboral y la tasa de ocupación en el sector informal.

La tasa de informalidad laboral corresponde a la proporción de la población ocupada que comprende a los laboralmente vulnerables por el tipo de unidad económica para la que trabajan y aquellos otros cuyo vínculo laboral no es reconocido por su fuente de trabajo. Además incluye a los ocupados en el servicio doméstico remunerado sin seguridad social, trabajadores no remunerados, trabajadores subordinados y remunerados que laboran sin la protección de la seguridad social (INEGI, 2013).

De acuerdo con los datos disponibles, durante el periodo 2008-2013 la tasa de informalidad laboral ha resultado en promedio en poco más de $59 \%$, en el año 2012 fue donde se obtuvo una mayor cantidad de personas laborando en este sector (59.85\%). Lo anterior permite reflexionar que dentro del mercado laboral mexicano, un poco más de mitad de la población ocupada se encuentra en condiciones desfavorables, sin acceso a seguridad social e insertos en unidades económicas que no aseguran la subsistencia del trabajador (véase la Figura 6) (INEGI, 2013).

La tasa de ocupación en el sector informal por definición corresponde a la población ocupada que trabaja para las unidades económicas que funcionan a partir de los recursos del hogar, pero sin constituirse como una empresa, de modo que los ingresos, materiales y equipos que se utilizan para el negocio no son independientes y/o distinguibles de los del propio hogar (INEGI, 2013). Para México, esta tasa abarca cerca una tercera parte de la población ocupada total en el país. De 2008 a 2013 se tuvo un crecimiento de 27.4 a $28.3 \%$ (véase la Figura 6).

La conducta de estos indicadores muestra un incremento en la ocupación de este sector. La dinámica visibiliza que una mayor parte de la población recurre a actividades informales como un medio para la obtención de ingresos. A pesar 


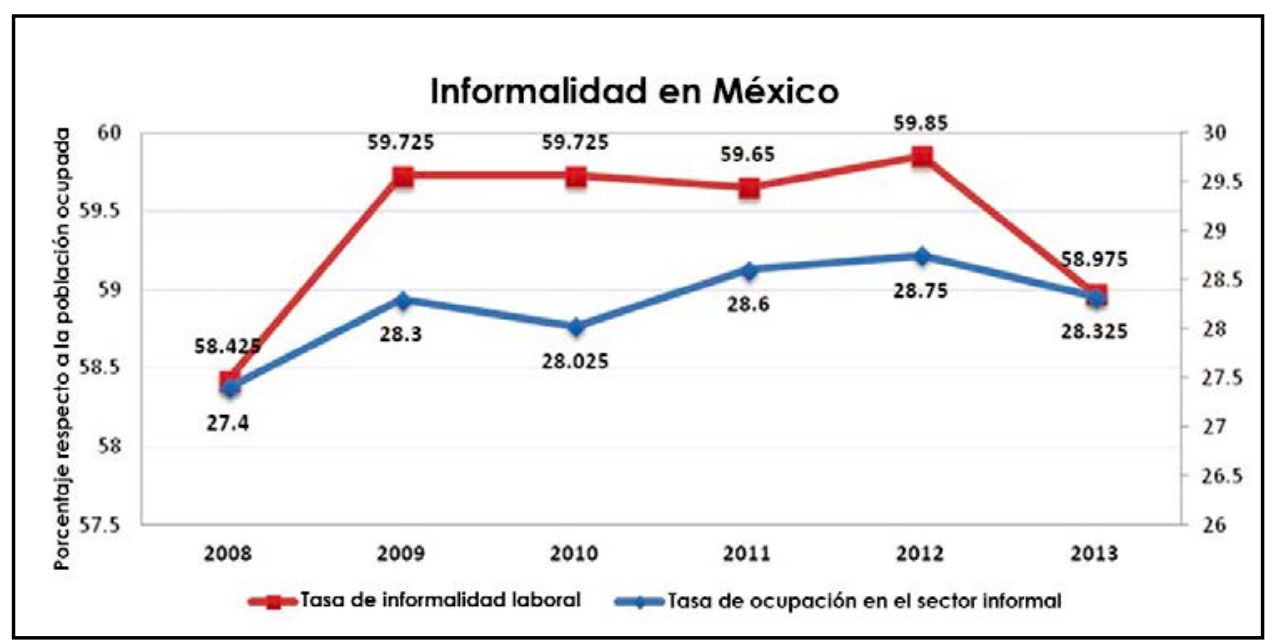

Figura 6. Tasa de informalidad laboral y de ocupación en el sector informal de México en el lapso 2003-2008. Elaboración propia con datos de la ENOE (INEGI, 2013).

de los esfuerzos por parte del gobierno mexicano para alinear a una mayor parte de la población en actividades formales, el esfuerzo ha sido insuficiente y los resultados poco exitosos. Es importante señalar que las condiciones mismas de la dinámica del mercado de trabajo mexicano incentivan una mayor participación en actividades informales, mismas que denotan malas condiciones laborales para la población.

\section{CONCLUSIONES}

El panorama presentado en este artículo muestra la fuerte tendencia que tiene el mercado de trabajo mexicano a la precarización del empleo. Se configura así un espacio laboral en el que predominan las unidades productivas centradas en el hogar y la

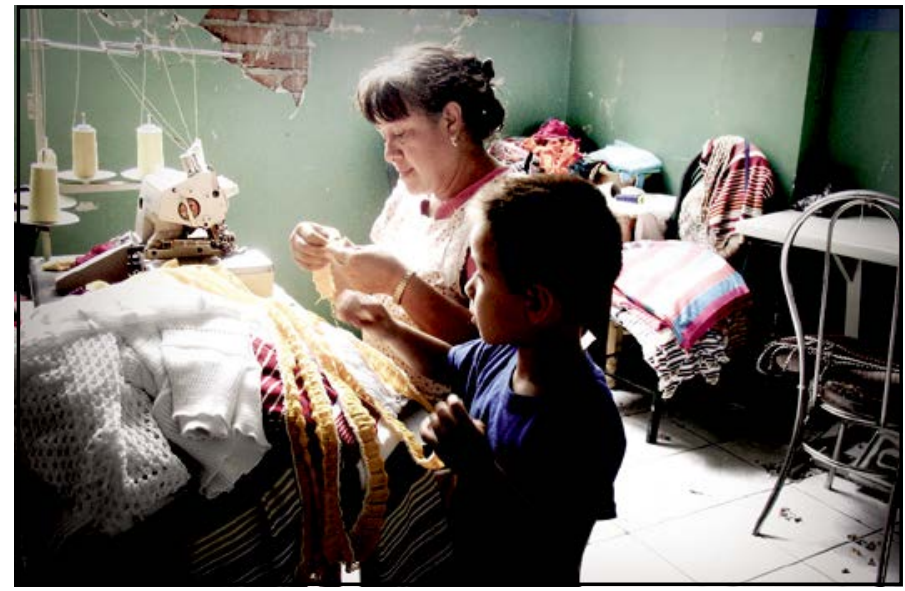

Figura 7. El trabajo dentro del taller familiar. Fotografía tomada por Dolly Ortiz. informalidad. Por ello se hace patente la necesidad de generar estrategias de gobierno y políticas públicas que pongan atención en estos espacios. Ejemplo de esto son los procesos de formalización desarrollados en Brasil, las cuales han logrado aumentos considerables en cuanto a los índices de trabajo protegido (Véras de Oliveira y Mota Braga, 2014). Las políticas brasileñas aumentaron los sistemas de vigilancia sobre las empresas, al tiempo que simplificaron los requisitos para el registro de los trabajadores.

El ITLP muestra la insuficiencia del salario, expresada por los pobres incrementos que ha sufrido en los últimos años. El trabajo que genera pobreza se vincula a la necesidad que tienen, aun los ocupados, de buscar ocupaciones alternativas para complementar sus ingresos. Este factor finalmente

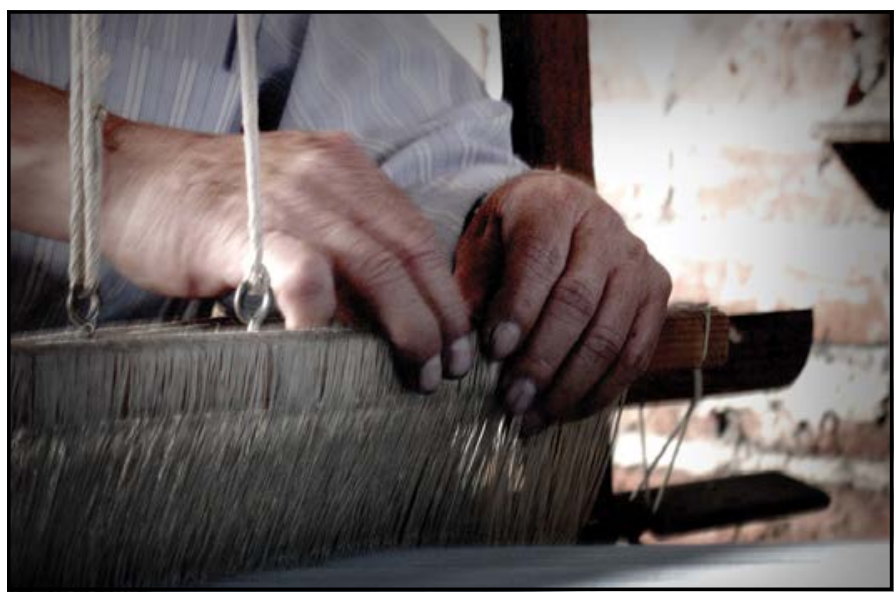

Figura 8. La ocupación de tejedor. Fotografía tomada por Dolly Ortiz. 
incide en el incremento de los trabajos informales $y$, por tanto, funciona como un lastre para el resto de la economía. Derivado de todo esto, una política que incentive salarios suficientes es fundamental para la solución de los problemas derivados del mercado de trabajo. El reto que se plantea es buscar las formas tanto sociales como legales que permitan elevar la calidad del empleo y la vida de los trabajadores mexicanos, a partir de diagnósticos que reflejen la realidad de nuestro mercado laboral.

LITERATURA CITADA

- CUEVAS RODRÍGUEZ, E. et al. Micronegocios y autoempleo en México. Un análisis empírico con datos de la Encuesta Nacional de Micronegocios. Carta Económica Regional, 17(94): 31-42, 2005

- De la GarzA TOledO, E. y NEFFA, J. C. (Comps.), Trabajo y modelos productivos en América Latina: Argentina, Brasil, Colombia, México y Venezuela luego de la crisis del modo de desarrollo neoliberal [Colección Grupos de Trabajo]. Buenos Aires, Argentina: CLACSO, 2010.

- HART, K. The idea of the economy: six modern dissenters. En R. FRIEDLAND y A. F. ROBERTSON (Eds.), Beyond the marketplace, rethinking economy and society (pp. 137-160). New York, US: Aldine de Gruyter, 1990.

- INEGI (INSTITUTO NACIONAL DE ESTADÍSTICA Y GEOGRAFíA) Encuesta Nacional de Micronegocios 2012. México: Autor, 2012.

- INEGI (INSTITUTO NACIONAL DE ESTADÍSTICA Y GEOGRAFíA) Encuesta Nacional de Ocupación y Empleo. Series de información trimestral de 2008 a 2013. México: Autor, 2013.

- MAZA díAZ CORTÉS, O. M. Las ventas multinivel. En M. E. PACHECO GÓMEZ MUÑOZ, E. DE LA GARZA TOLEDO y L.
RAYGADAS (Eds.), Trabajos atípicos y precarización del empleo (p. 299). D. F., México: El Colegio de México, 2011.

- pacheco gómez muñoz, M. E. Ciudad de México, heterogénea y desigual: un estudio sobre el mercado de trabajo. D. F., México: El Colegio de México, 2004.

- PACHeCO GÓMEZ MUÑOZ, M. E. et al. Trabajos atípicos y precarización del empleo. D. F., México: El Colegio de México, 2011.

\section{De páginas electrónicas}

- coneval ICONSEjo nacional de eVAluación de la POLÍTICA DE DESARROLLO SOCIAL) Resultados del Índice de Tendencia Laboral de la Pobreza (ITLP) al segundo trimestre de 2014, s.f. Recuperado el 1 de julio de 2014, de http://www. coneval.gob.mx/Medicion/Paginas/ITLP_ITLP_IS.aspx

- VÉRAS DE OLIVEIRA, R. y MOTA BRAGA, B. Território comercial de Toritama: persistência e metamorfoses da informalidade. POLITICA \& TRABALHO. Revista de Ciencias Sociais, 41, 193-225, 2014. Recuperado de http://periodicos.ufpb.br/index.php/ politicaetrabalho/article/viewFile/21820/12655 